\title{
Fast Analysis of Photonic Crystal Structures With Mixed-Order Prism Macroelements
}

\author{
Dimitrios I. Karatzidis, Traianos V. Yioultsis, and Emmanouil E. Kriezis, Member, IEEE
}

\begin{abstract}
In this paper, a new concept of a mixed-order prism macroelement is introduced for the analysis of photonic crystal slabs and waveguiding structures. The mixed-order concept used here implies arbitrary orders of variation towards different directions and it differs essentially from the well-known mixed-order approximation that is an integral part of every Whitney element. The reduction of degrees of freedom, achieved by the employment of such elements, facilitates the solution of the resulting eigenvalue equation. Thus, an efficient 3-D finite-element analysis tool is developed to calculate the band structure of both symmetric and asymmetric photonic crystal slabs. Dispersion relation results for two different types of photonic crystal waveguides are also presented, to further verify the method.
\end{abstract}

Index Terms-Finite-element method (FEM), periodic structures, photonic crystal slabs, photonic crystal waveguides.

\section{INTRODUCTION}

$\mathbf{T}$ HE ability of photonic crystals [1], [2] to control light propagation has attracted significant research attention in recent years. Evolution in technology and improvement in theoretical and numerical methods provided further insight into their properties, leading to new device concepts and designs, such as photonic crystal slabs [3], [4], waveguides [5]-[8], Y-junctions [9], resonant cavities [10]-[12], couplers [13], filters [14], and many more. In particular, photonic crystal slabs and waveguides are essential building blocks for photonic crystal circuits and band information of such structures is of great importance for design purposes. Optical circuits, based on photonic crystal slabs, provide lateral confinement of light by a 2-D lattice, whereas vertical confinement is provided by the refractive index contrast of the layered structure. Although some of the benefits of full 3-D photonic crystals are lost, the relative ease of fabrication seems to be an offset for such a loss.

The correct description of photonic crystal properties requires the solutions of the full vectorial Maxwell's equations. Thus, numerical modeling is crucial, since no analytical approximation is possible and the fabrication is a costly and time-consuming process. Among the most widely used and powerful computational techniques are the planewave expansion (PWE) method [15]-[17] and the finite-difference

Manuscript received July 27, 2007; revised January 29, 2007. Published August 29, 2008 (projected).

The authors are with the Telecommunications Division, Department of Electrical and Computer Engineering, Aristotle University of Thessaloniki, Thessaloniki GR-54124, Greece (e-mail: dkaratzid@yahoo.com; traianos@auth.gr; mkriezis@auth.gr).

Color versions of one or more of the figures in this paper are available online at http://ieeexplore.ieee.org.

Digital Object Identifier 10.1109/JLT.2008.922209 time-domain (FDTD) method [18]-[22]. The PWE method is based on a time-harmonic planewave eigenmode decomposition and a Fourier series expansion of the dielectric permittivity. It is straightforward, easy to implement, and most widespread technique for the calculation of photonic crystal dispersion diagrams. A critical issue, however, is related to the convergence dependence on the number of planewaves employed. An alternative approach for photonic band calculations can be found in [23], where the magnetic field is expanded in terms of guided modes. The FDTD method is another popular method for photonic crystal calculations, including the derivation of dispersion diagrams [18], [20]. It has been proved accurate and it is relatively easy to implement. However, it exhibits some inherent limitations, such as the staircase approximation, which renders modeling of fine features difficult and requires long calculation times, due to the excessive number of necessary time steps. Another drawback, related to the construction of dispersion diagrams, is the difficulty in resolving the resonant peaks, which in many cases would require the employment of advanced digital signal processing techniques. In addition, it is generally difficult to obtain the mode profiles in a single FDTD run. On the other hand, the finite-element method (FEM) has also proven to be a flexible and efficient numerical tool in photonic structure modeling in 2-D [24], [25] or 3-D [26], [27], though its application in planar photonic crystal dispersion calculations [28] is not very widespread. The use of FEM would be particularly advantageous due to the sparsity of the resulting matrices and the inherently accurate modeling of sharp material discontinuities or fine geometric features. However, for this particular class of problems in planar photonic crystal geometries, a classical tetrahedral or hexahedral element implementation does not seem to be optimal, since it would require an enormous number of degrees of freedom, possibly rendering the application of FEM prohibitive. If, on the other hand, one somehow manages to take into account the planar configuration of the slab, possibly by means of properly modeling field variations on the direction normal to the plane of periodicity, the number of degrees of freedom could be significantly reduced.

In this paper, a new prismatic vector finite element is presented that is specially designed to deal with such kind of planar photonic crystal structures. The approach followed herein is essentially different from that in [28], where a second-order prism element was employed. The prism element used here incorporates different orders of variations along different directions. It can be considered a macroelement, in that a higher order variation is assumed only towards its axis, to capture moderate field variations in the direction normal to the slab. Since the geometric configuration of a planar structure does not change within 
a specific layer, only one element per layer will be necessary. On the other hand, possibly complex geometric features on the plane of periodicity can be properly modeled by first-order elements and a sufficiently fine mesh. Such a setting enables easy geometric modeling, via 2-D mesh generation and, most importantly, a significant reduction in degrees of freedom, compared to classical tetrahedral or hexahedral elements. Though the extension of the proposed macroelements to include higher order variations in the plane of periodicity is feasible, we have chosen to use first-order variation in the plane of periodicity (where finer features are accounted for by proper mesh refinement). On the other hand, in planar geometries, the higher order in the third dimension is considered more critical in order to capture the field variation with a single macroelement per material layer. Additionally, the proposed scheme maintains reasonable simplicity, whereas increasing the order of variation both in the planar and vertical directions will substantially increase the complexity of the analysis and implementation.

In Section II, the element construction procedure is briefly discussed and the vector basis functions are derived. The finite-element formulation with periodic boundary conditions is briefly outlined and, in Section III, it is applied to obtain the band information of commonly encountered photonic crystal slabs. To further confirm our approach, the dispersion relations for two types of waveguides are calculated: a linear-defect waveguide in a finite-sized rod arrangement and a waveguide in a triangular lattice of air holes. In addition, indicative mode field profiles are provided.

\section{FORMULATION}

\section{A. Development of Third-Order Vector Prism Macroelements}

The macroelement that is presented in this section will be constructed on a usual geometric prism element; however, the degree of approximation will not be the same along different directions. The idea behind its design is that in a layered structure, the electromagnetic field exhibits a moderate variation along the vertical direction, while more intense variations are anticipated in the lateral plane due to the existence of the crystal lattice. It is, therefore, reasonable to use long prism macroelements that exhibit the simplest (first) order of variation along the lateral directions, and a sufficiently high order along their axis, thus being able to account for the field variations in the vertical direction using a single element per layer. On the other hand, lateral variations are mainly associated to the lattice pattern and are expected to be sufficiently modeled by a fine mesh of first-order finite elements. Usage of a higher order approximation in the lateral plane would substantially increase the number of degrees of freedom, without contributing much to an accuracy improvement. Therefore, such a macroelement will be appropriate to capture the physics of the problem, while requiring only 2-D preprocessing and mesh generation. It is worth to mention that this approach has already been successfully applied in the optimization of microstrip antennas [29].

The formation of the macroelement is performed by mathematically imposing the basic principles that are essential to any Whitney element with tangential continuity. First, appropriate degrees of freedom have to be chosen, based on tangential projections on the element edges or faces and, most notably, the inclusion condition, i.e., a proper modeling of the curl-free fields should be preserved in the discrete level as well. Generally, under these assumptions, a Whitney element turns out to satisfy the so-called mixed-order property, that is the variation of any field component along its direction is one order less than in the respective normal plane. The mixed-order concept introduced here is different, in the sense that a third-order longitudinal variation is adopted and a first-order planar variation is maintained, in other words, we have different variations in the same element. Of course, separate field components will comply with the usual mixed-order principle, in consistency with both the physics of the problem under study and the correct approximation of the curl operator nullspace, without violation of the fundamental mixed-order principle. The degrees of freedom are defined by proper weighted tangential projections on the element's edges and faces and the enforcement of the inclusion condition, combined with the decoupling conditions, finally lead to the derivation of vector basis functions.

More specifically, the transverse variations are expressed via the simplex coordinates, while a normalized axial coordinate $-1 \leq \zeta_{0} \leq 1$ for longitudinal variations is used. According to the principles laid out in [30], a general field expression for a third-order element is

$$
\begin{aligned}
& \mathbf{F}=\hat{\mathbf{x}}\left(y, z, z y, z^{2}, z^{2} y, z^{3}, z^{3} y\right) \\
&+\hat{\mathbf{y}}\left(x, z, z x, z^{2}, z^{2} x, z^{3}, z^{3} x\right) \\
&+\hat{\mathbf{z}}\left(x, y, z, z x, z y, z^{2}, z^{2} x, z^{2} y\right) .
\end{aligned}
$$

This expansion includes third-order variations along the $z$-axis only, but the usual mixed-order principle continues to hold, since transverse field components have such variations, while the axial component has only first- and second-order variations. Considering (1) and following a systematic approach [30], we find that the required number of degrees of freedom is 21 . We notice that a prismatic Whitney element of second- or third-order variations to all directions would require 36 and 89 degrees of freedom, respectively. Six degrees of freedom of the form

$$
F_{i j}=\int_{(i)}^{(j)} \mathbf{F} \cdot \mathrm{dl}
$$

are assigned to the six edges of the triangular faces. Three degrees of freedom on each one of the three vertical edges are defined as weighted tangential projections of the form

$$
\begin{aligned}
F_{i j}^{i i} & =\int_{(i)}^{(j)} \mathbf{F} \cdot \hat{\mathbf{z}} \frac{\zeta_{0}\left(\zeta_{0}-1\right)}{2} d z \\
F_{i j}^{j j} & =\int_{(i)}^{(j)} \mathbf{F} \cdot \hat{\mathbf{z}} \frac{\zeta_{0}\left(\zeta_{0}+1\right)}{2} d z \\
F_{i j}^{i j} & =\int_{(i)}^{(j)} \mathbf{F} \cdot \hat{\mathbf{z}}\left(1-\zeta_{0}\right)\left(1+\zeta_{0}\right) d z
\end{aligned}
$$

where the weights are the basis functions of the second-order nodal element. The derivation of the remaining six face degrees of freedom, two on each one of the three faces, is not trivial but 


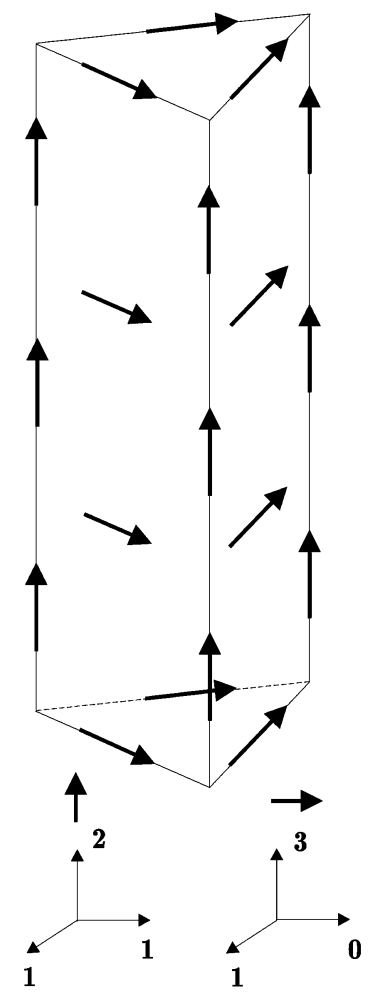

Fig. 1. Third-order prism macroelement: degrees of freedom and order of variations for the axial and a transverse component.

we can construct them in a straightforward manner by considering the surface integral on a face $\{i, j, k, l\}$

$$
\iint_{(i, j, k, l)} \nabla \times\left(\frac{\zeta_{0}\left(\zeta_{0}-1\right)}{2} \mathbf{F}\right) \cdot \hat{\mathbf{n}} d s .
$$

By applying Stoke's theorem and appropriate vector algebra identities, we can express the degree of freedom for the normally continuous field $\nabla \times \mathbf{F}$, in terms of the already defined edge degrees of freedom. Thus, the face degrees of freedom are

$$
\begin{aligned}
& F_{i j k l}^{1}=\iint_{(i, j, k, l)}\left(\zeta_{0}-\frac{1}{2}\right)(\mathbf{F} \times \hat{\mathbf{n}}) \cdot \nabla \zeta_{0} d s \\
& F_{i j k l}^{2}=\iint_{(i, j, k, l)}\left(\zeta_{0}+\frac{1}{2}\right)(\mathbf{F} \times \hat{\mathbf{n}}) \cdot \nabla \zeta_{0} d s .
\end{aligned}
$$

In Fig. 1, placement of the above 21 degrees of freedom and the respective orders of field variations are shown.

The coefficients for the vector basis functions are obtained by imposing the proper decoupling conditions and the constraints dictated by the inclusion condition. Although the latter one requires a strenuous algebraic manipulation, it results in quite simple constraints, i.e., the highest order terms in the field expansion should appear with opposite signs. The basis functions for an edge $[i, j]$ of a triangular face will be given by

$$
\begin{aligned}
\mathbf{w}_{i j}= & \left(-\frac{5}{4} \zeta_{0}^{3}+\frac{3}{4} \zeta_{0}^{2}+\frac{3}{4} \zeta_{0}-\frac{1}{4}\right) \zeta_{i} \nabla \zeta_{j} \\
& +\left(\frac{5}{4} \zeta_{0}^{3}-\frac{3}{4} \zeta_{0}^{2}-\frac{3}{4} \zeta_{0}+\frac{1}{4}\right) \zeta_{j} \nabla \zeta_{i}, \quad(z=0) \\
\mathbf{w}_{i j}= & \left(\frac{5}{4} \zeta_{0}^{3}+\frac{3}{4} \zeta_{0}^{2}-\frac{3}{4} \zeta_{0}-\frac{1}{4}\right) \zeta_{i} \nabla \zeta_{j} \\
& -\left(\frac{5}{4} \zeta_{0}^{3}+\frac{3}{4} \zeta_{0}^{2}-\frac{3}{4} \zeta_{0}-\frac{1}{4}\right) \zeta_{j} \nabla \zeta_{i}, \quad(z=h)
\end{aligned}
$$

where $h$ is the prism height. The expressions for face basis functions are

$$
\begin{aligned}
\mathbf{w}_{i j k l}^{1}= & \left(-\frac{15}{8} \zeta_{0}^{3}+\frac{3}{4} \zeta_{0}^{2}+\frac{15}{8} \zeta_{0}-\frac{3}{4}\right) \zeta_{i} \nabla \zeta_{j} \\
& +\left(\frac{15}{8} \zeta_{0}^{3}-\frac{3}{4} \zeta_{0}^{2}-\frac{15}{8} \zeta_{0}+\frac{3}{4}\right) \zeta_{j} \nabla \zeta_{i} \\
\mathbf{w}_{i j k l}^{2}= & \left(-\frac{15}{8} \zeta_{0}^{3}-\frac{3}{4} \zeta_{0}^{2}+\frac{15}{8} \zeta_{0}+\frac{3}{4}\right) \zeta_{i} \nabla \zeta_{j} \\
& +\left(\frac{15}{8} \zeta_{0}^{3}+\frac{3}{4} \zeta_{0}^{2}-\frac{15}{8} \zeta_{0}-\frac{3}{4}\right) \zeta_{j} \nabla \zeta_{i}
\end{aligned}
$$

and for the vertical edges

$$
\begin{aligned}
\mathbf{w}_{i j}^{i i} & =\left(\frac{15}{4} \zeta_{0}^{2}-\frac{3}{2} \zeta_{0}-\frac{3}{4}\right) \zeta_{i} \nabla \zeta_{0} \\
\mathbf{w}_{i j}^{j j} & =\left(\frac{15}{4} \zeta_{0}^{2}+\frac{3}{2} \zeta_{0}-\frac{3}{4}\right) \zeta_{i} \nabla \zeta_{0} \\
\mathbf{w}_{i j}^{i j} & =\left(-\frac{15}{8} \zeta_{0}^{2}+\frac{9}{8}\right) \zeta_{i} \nabla \zeta_{0} .
\end{aligned}
$$

\section{B. Finite-Element Analysis}

A generic problem geometry is illustrated in Fig. 2. The computational domain is an appropriate unit cell of the crystal lattice, consisting of three layers (substrate/guiding layer/superstratum). Periodicity is along $x$ and/or $y$ directions and a finite height in the $z$ direction is assumed. A FEM description of the problem geometry is obtained by subdividing the unit cell volume into small prism macroelements. The height of the macroelements is adjusted so that a single macroelement per layer is used. The Galerkin formulation of the vector Helmholtz eigenvalue equation for the electric field is given by

$$
\begin{array}{r}
\iiint_{\Omega} \nabla \times \mathbf{w}_{i}^{*} \cdot \mu_{r}^{-1} \nabla \times \mathbf{w}_{j} d \Omega-k_{0}^{2} \iiint_{\Omega} \mathbf{w}_{i}^{*} \cdot \epsilon_{r} \mathbf{w}_{j} d \Omega \\
=\oiint_{\partial \Omega} \mathbf{w}_{i}^{*} \times \mu_{r}^{-1} \nabla \times \mathbf{w}_{j} \cdot d \mathbf{S} .
\end{array}
$$

The surface integral term in (9) vanishes on boundaries that correspond to perfect electric conductors (PECs) or periodic boundaries [31].

The 2-D mesh generation greatly facilitates a consistent, with respect to periodicity, grid. Thus, we can define identical degrees of freedom on a pair of periodic surfaces $\left(m-m^{\prime}, n-\right.$ $n^{\prime}$ ) and by use of Bloch's theorem, we impose the periodic boundary conditions (PBCs) on the corresponding basis functions, i.e.,

$$
\mathbf{w}_{m^{\prime}}=\exp (-j \mathbf{k} \cdot \mathbf{a}) \mathbf{w}_{m} \quad \mathbf{w}_{n^{\prime}}=\exp (-j \mathbf{k} \cdot \mathbf{b}) \mathbf{w}_{n} .
$$

On the top and bottom surfaces, the mesh is truncated by placing PEC boundary conditions at an appropriate distance, where the mode profile is expected to have sufficiently decayed. This distance can be easily estimated in a preprocessing step by examining the mode profiles of the unpatterned slab, by a simple analytical calculation. Once a rough estimate of the necessary distance is available, one can possibly decide on the number of prismatic element layers that are required or the appropriate 


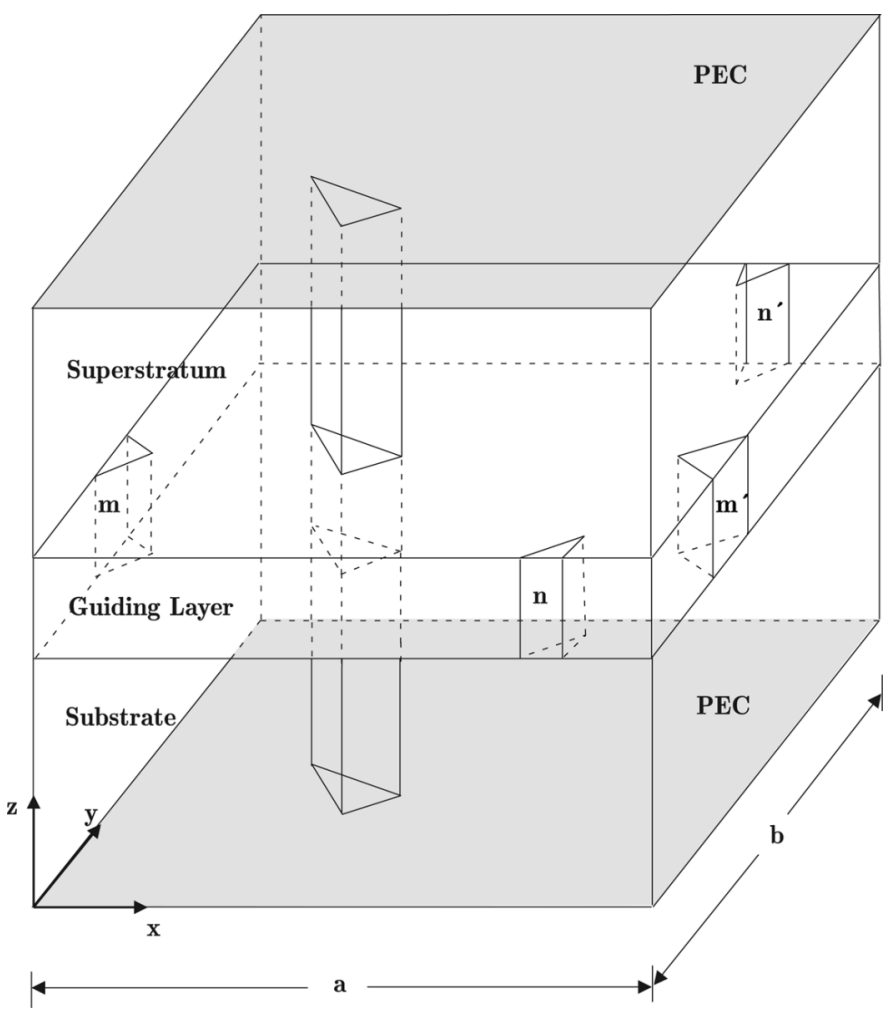

Fig. 2. Unit cell geometry and boundary conditions.

order of variation along the macroelement axis. Finally, a generalized eigenvalue equation of the form $\mathbf{A x}=\lambda \mathbf{B x}$ is obtained. The PEC boundary conditions guarantee the symmetry of the resulting matrices, whereas enforcement of (10) results in complex matrix elements, corresponding to degrees of freedom on periodic surfaces. The MATLAB routine sptarn is used for the solution of the eigenvalue problem. The eigenvalue search interval can be optimally determined, saving considerable computational time. Moreover, the introduction of a higher order variation on the vertical dimension only contributes to a dramatic reduction of degrees of freedom. Application of open boundary conditions is also possible. The widespread PML absorbing boundary condition is preferred in other computational methods (like FDTD) but it is not the primary choice in frequency-domain FEM formulations, since it is known to severely affect the matrix conditioning, resulting in poor performance. First-order absorbing boundary conditions (ABC) are usually preferable for mesh truncation in FEM, although the resulting matrices are complex nonsymmetric, and ill-conditioned, and poor performance of the eigenvalue solver is observed as well. However, it is clear that application of an $\mathrm{ABC}$ is not considered critical in the class of problems dealt with in this paper (i.e., band diagram calculations). Furthermore, the proposed approach is not restricted in the context of a generalized eigenvalue problem. For the wide class of excitation-driven problems, where no eigenvalue solver is involved, the mixed-order prism macroelement is expected to be equally efficient, and the introduction of an $\mathrm{ABC}$ or even the PML is generally considered to be acceptable. Such an implementation will allow its application in photonic crystal device modeling, with the device being excited by arbitrary impinging waves.

\section{ANALYSIS OF PHOTONIC CRYSTAL StRUCTURES}

\section{A. Slabs}

We consider first a square lattice of dielectric rods suspended in air [4] with height $2 a$ and radius $0.2 a$, where $a$ is the lattice constant. The relative dielectric permittivity of the rods is 12 . PEC boundaries are placed at a distance of $2 a$ away from the top and bottom surfaces of the rods. Band diagrams for the three lowest order even (TE-like) and odd (TM-like) bands are shown in Fig. 3(a) and (b), respectively. The number of prism elements is 1464 , which corresponds to 8052 degrees of freedom. Results are compared with those obtained using the PWE method [17] by means of the open-source MIT Photonic-Bands Package (MPB) [32] and very good agreement is observed. A convergence plot, showing computed values of normalized frequency (at point $X$ ), with respect to the number of degrees of freedom is given in Fig. 3(c). The maximum relative error for almost all bands is less than $1 \%$ and only for a few values of the third even band it is slightly higher but still less than $2 \%$. In Fig. 4, a profile of the $E_{z}$ electric field component is shown for the lowest order odd band at the $M$ symmetry point.

We also have to note that in order to calculate the higher order bands we have to set a slightly larger search interval in the eigenvalue solver and further select an appropriate position for the PEC boundary. Since higher order bands (higher frequencies) are more localized in the slab region, they would allow bringing the PEC boundary closer to the slab. This is also necessary, as a reduced macroelement height is needed in order to capture the field variations just above and below the slab. This would compromise the accuracy for the lower order bands and thus suggests two separate runs for the higher and lower bands, respectively. The above strategy still allows for a single layer of macroelements above and below the slab; alternatively, one can use two layers of macroelements in order to get the results in a single run at the expense of an increased number of unknowns.

A triangular lattice of air voids in a dielectric slab is considered next [4]. A slab thickness of $0.6 a$ and a hole radius is $0.45 a$ are chosen, with the background regions above and below the guiding layer being occupied by a uniform dielectric material. The dielectric permittivity of the substrate is 2 and the guiding layer permittivity is 12 . The substrate and superstratum are extended to a distance of $2 a$, where PEC termination is used. A total of 1752 elements with 9636 degrees of freedom were involved in the band structure calculations. The resulting dispersion diagram is shown in Fig. 5, where the curves from the MPB package are depicted as well. A maximum absolute error of $1 \%$ is recorded in most cases, with few exceptions approaching $2 \%$.

We now turn to the case where the layers above and below the slab are of different refractive index. Such an asymmetric photonic crystal slab still exhibits band gap effects [33], though the guided modes can no longer be classified as even or odd, due to the breaking of mirror symmetry. Fig. 6 depicts the dispersion curves for the three lowest order guided modes of a triangular lattice of air holes in an asymmetric slab. The air holes with radius $0.3 a$ are etched only through the guiding layer of thickness $0.4 a$. The guiding layer refractive index is 3.6, the substrate index is 1.5 , and no material is placed on top [33]. A PEC boundary at a height of $3.5 a$ truncates the upper air layer, 


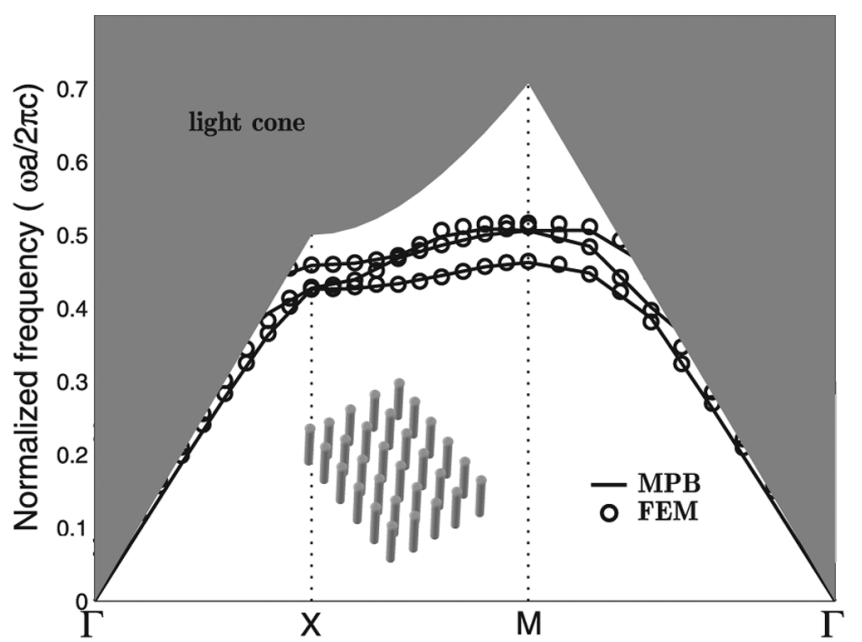

(a)

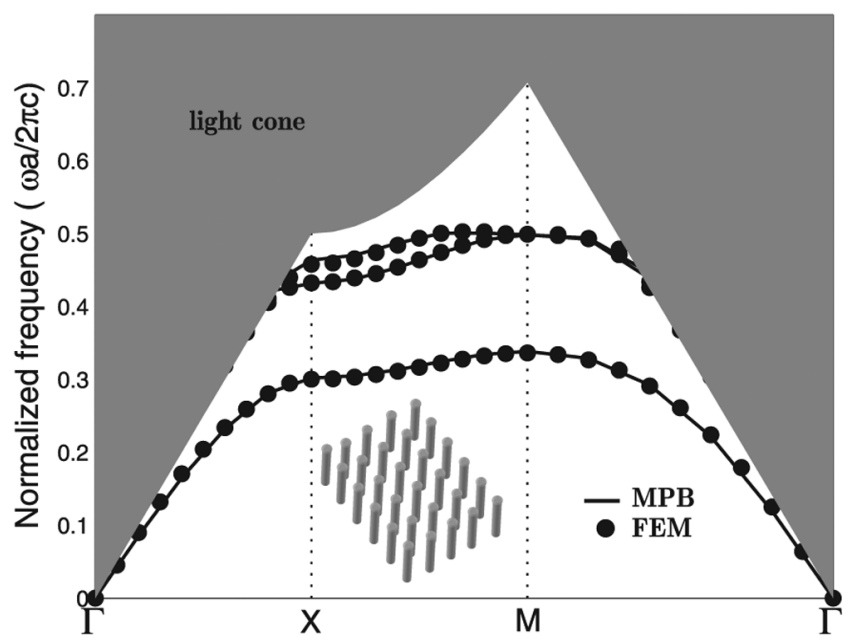

(b)

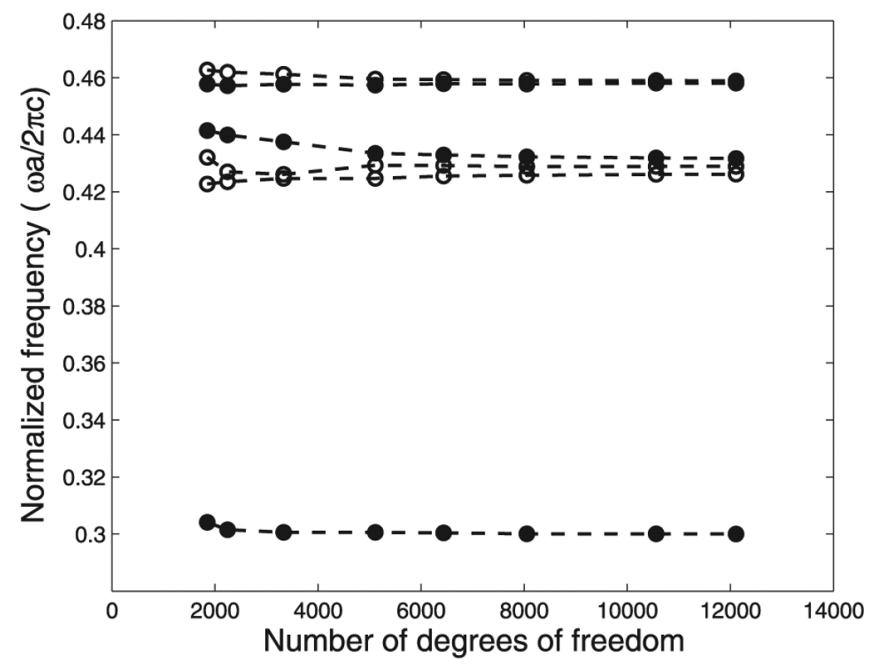

Fig. 3. Band diagram for the three lowest order modes for rods arranged in a square lattice and suspended in air: (a) TE-like and (b) TM-like. (c) Convergence of results (point $X$ ) with respect to the number of degrees of freedom, for the three lowest order TE-like modes (open circles) and TM-like modes (solid circles).

whereas the placement of the PEC boundary at a lower distance of $1.5 a$ is sufficient to capture the field variations in the structure. With a total of 1674 elements and 9207 degrees of freedom,

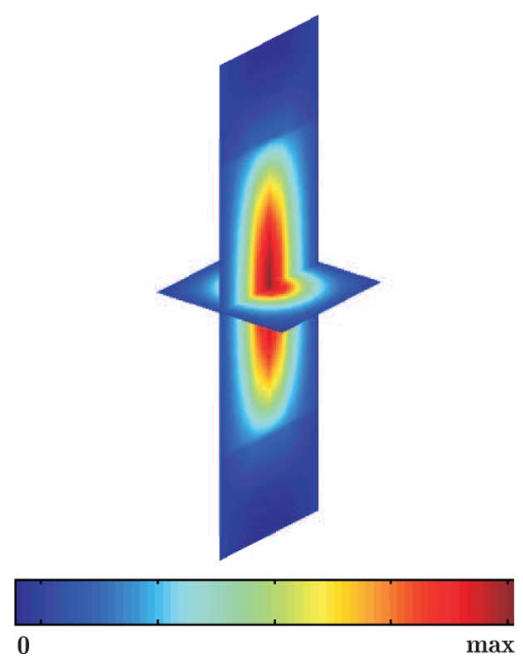

Fig. 4. Real part of $E_{z}$ component for the square lattice of rods. The profile corresponds to the first odd band at the $\mathrm{M}$ symmetry point.

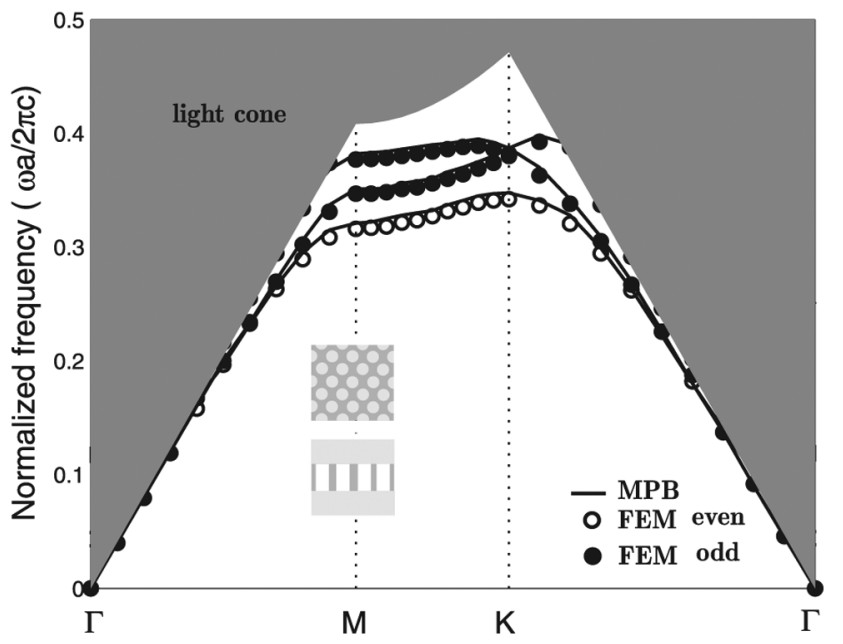

Fig. 5. Band diagram for the triangular lattice of air holes in a high-index slab, surrounded by an unpatterned lower index dielectric.

the relative error for most values is less than $1 \%$, while a small number of values exhibit slightly higher values but still less than $2 \%$. In all cases, it has been verified that an increase in mesh density reduces the overall relative error to well below $1 \%$.

Finally, we have assessed the computational efficiency (execution times) of the proposed method in comparison with both the MPB package and MIT Electromagnetic Equation Propagation (MEEP), an open source implementation of the 3-D FDTD method [34]. For the case of the rods arranged in a square lattice, the following computational times per wavevector were recorded in a high-end desktop computer: $5^{\prime} 53^{\prime \prime}$ for the proposed FEM technique (8052 degrees of freedom), $2^{\prime} 02^{\prime \prime}$ for the MPB code $(64 \times 64 \times 128$ planewaves $)$, and $6^{\prime} 07^{\prime \prime}$ for the MEEP solution (with a spatial resolution of 26 cells per lattice constant).

\section{B. Waveguides}

Having assessed the proposed methodology with photonic crystal slabs, we turn our attention to the more complex case of photonic crystal waveguides. As a first example we calculate the 


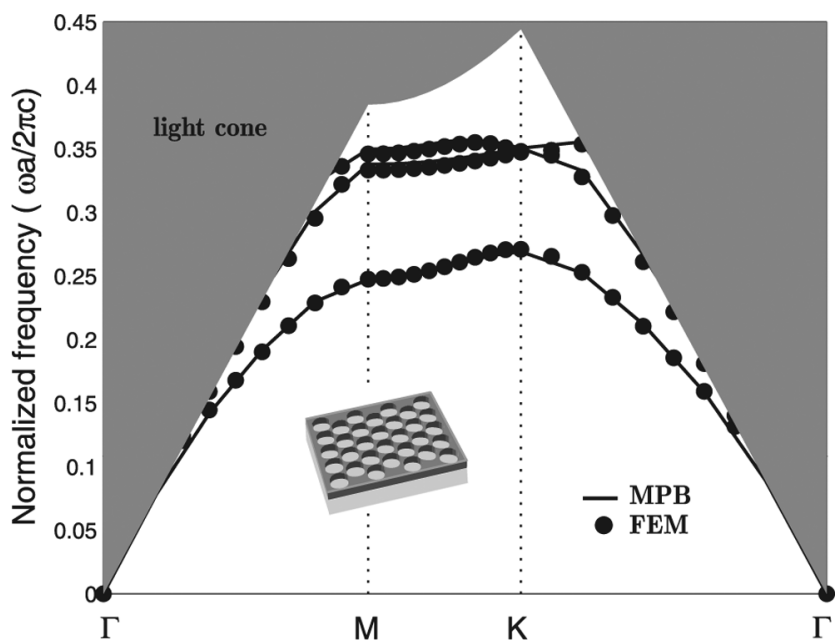

Fig. 6. Band structure of a triangular air-hole lattice, etched in a silicon-oninsulator (SOI) wafer.

dispersion relation for guided modes in a waveguide formed by decreasing the radii of dielectric rods in a single row. This defect is along the $\Gamma-X$ symmetry direction in a square lattice of rods, suspended in air [35]. Every single rod has a central segment of height equal to $2 a$ and permittivity of 12 , and extends above and below with a permittivity equal to 2 . The bulk radius is $0.2 a$ and the line defect radius is $0.14 a$. Since the photonic crystal symmetry is only broken in one direction, PBCs are applied perpendicular to the defect axis. PEC boundaries are used to truncate the computational domain in the lateral and vertical directions. The top and bottom boundaries are placed at a distance of $6 a$ from the central rod segment, while the lateral PEC boundaries are placed at a distance of four lattice constants at both sides. The number of elements is 2868 , resulting in a total of 15764 degrees of freedom. Fig. 7 shows the waveguide dispersion relation, where only one odd symmetry (TM-like) mode is present. The dispersion relation for a line defect radius of $0.12 a$ is also presented. The obtained results are in excellent agreement with those reported in the literature [35]. The computational unit cell distribution of the $E_{z}$ (TM-like mode) field component, in the cross section and in the plane that is perpendicular to the defect axis and bisects a row of rods, are shown in Fig. 8 .

A waveguide in a triangular lattice of air holes, etched in a slab, is examined next [36]. The waveguide is made by leaving out a single line of holes in the $\Gamma-K$ direction ( $W 1$ guide). The lattice parameters are as follows: slab thickness $0.6 a$, air hole radius $0.29 a$, and refractive index of the dielectric slab 3.4. Air is assumed at both sides of the slab. The PEC boundaries are placed at a distance of $1.8 a$ from the slab surfaces. PBCs are applied at planes perpendicular to the defect axis. PEC boundaries are also applied at a distance of five lattice constants at both sides. A total of 2844 elements were used with 15523 degrees of freedom. The projected band diagram is shown in Fig. 9. Two TE-like modes are found to exist in the bandgap, which are furthermore distinguished by their lateral even-odd symmetry. Excellent agreement with published results [36] has been confirmed as well.

The different kind of lateral symmetry for TE-like modes is clearly illustrated in Figs. 10 and 11. The computational effi-

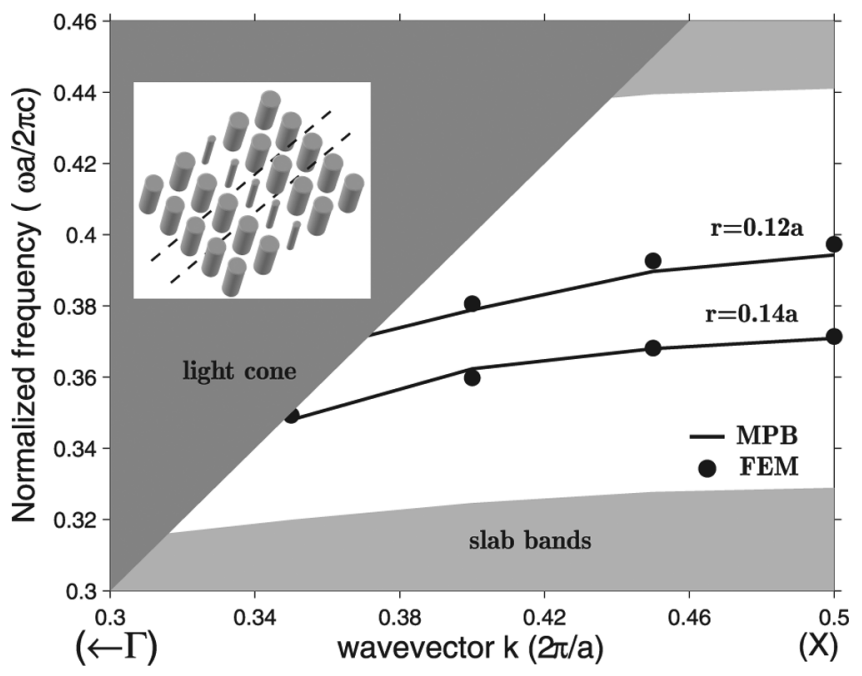

Fig. 7. Projected band diagram for the reduced-radius rod-slab waveguide. The odd symmetry (TM-like) guided modes for two defect rod radii are shown. The computational unit cell is marked with dashed lines in the inset.

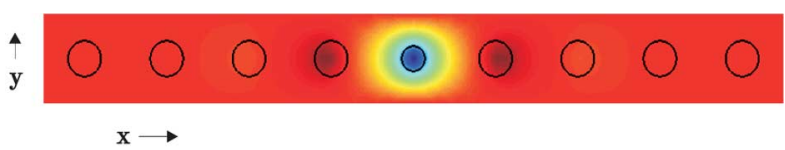

(a)

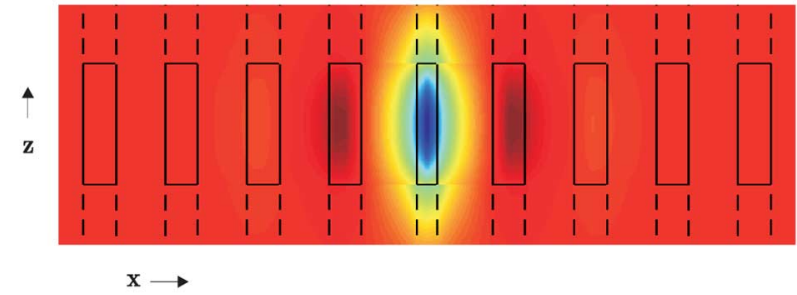

(b)

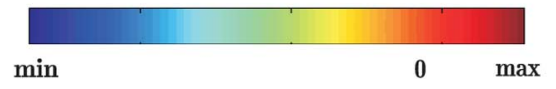

Fig. 8. Real part of $E_{z}$ for a single defect row waveguide (defect radius is $0.14 a$ ), in a rectangular lattice of rods at the $X$ point: (a) horizontal cross section and (b) vertical cross section. The solid line indicates the high-index material, whereas the dashed line represents the low-index material.

ciency is assessed also in the case of photonic crystal waveguides. For the case of the reduced-radius rod-slab waveguide, the computational times per wavevector were $10^{\prime} 15^{\prime \prime}$ for the proposed FEM technique (15 764 degrees of freedom), $11^{\prime} 05^{\prime \prime}$ for the MPB code $\left(80 \times 16 \times 80\right.$ planewaves), and $18^{\prime} 47^{\prime \prime}$ for the MEEP solution (with a spatial resolution of 14 cells per lattice constant).

\section{CONCLUSION}

We have developed a new fast 3-D FEM analysis tool for band diagram calculations of planar photonic crystal structures. Such a macroelement configuration enables the use of 2-D mesh generation for their geometric description, where the third dimension is accounted for, using a higher order variation. Its applicability and accuracy are thoroughly demonstrated in a series of examples, where the band diagrams of various kinds of photonic crystal slabs and waveguides are derived. A further exten- 


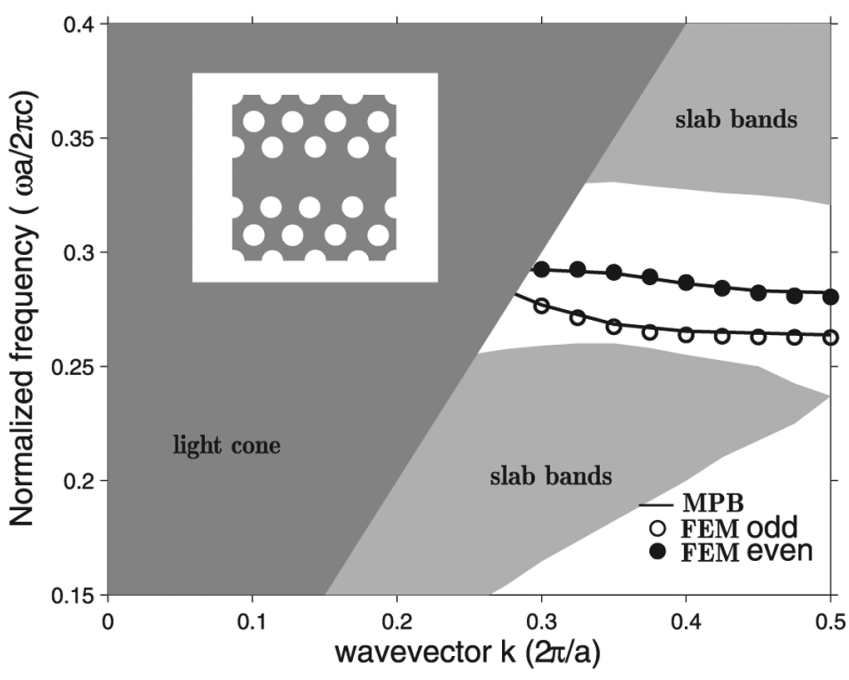

Fig. 9. Projected band diagram for the $W 1$ guide, formed in the air-suspended membrane of a triangular lattice. Even symmetry (TE-like) guided modes are shown. The even-odd distinction is based on the lateral field symmetry. The computational unit cell is marked with dashed lines in the inset.

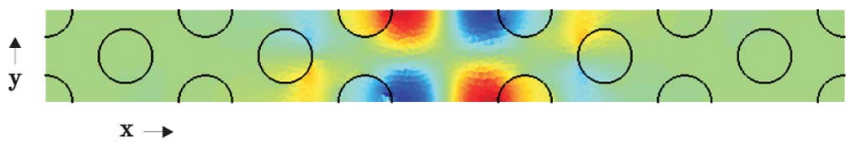

(a)

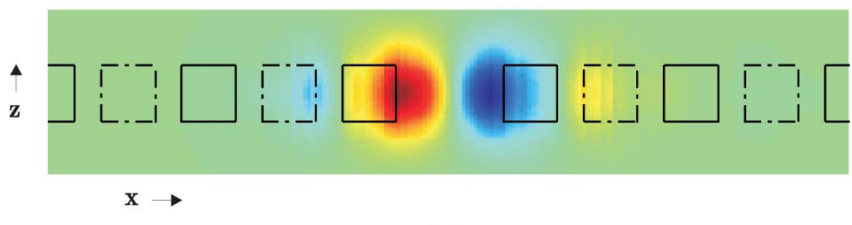

(b)

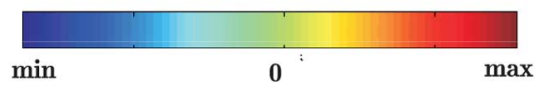

Fig. 10. Real part of $E_{y}$ for the TE laterally odd mode at $k=\pi / a$ : (a) midplane horizontal cross section and (b) vertical cross section at $y=a / 2$.

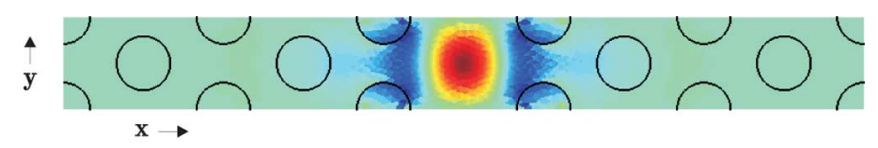

(a)

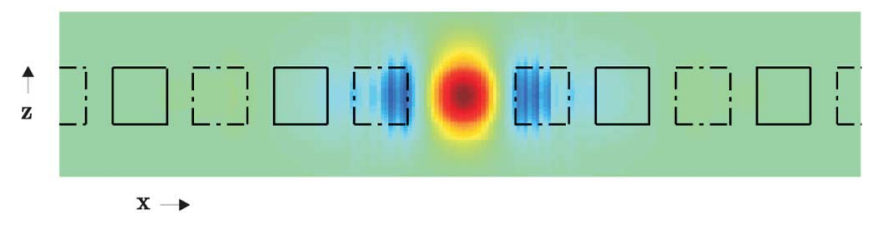

(b)

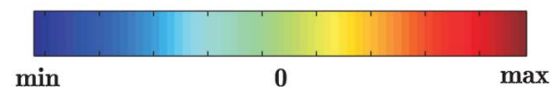

Fig. 11. Real part of $E_{y}$ for the TE laterally even mode at $k=\pi / a$ : (a) midplane horizontal cross section and (b) vertical cross section at $y=a$.

sion of this work is that, apart from calculating PBG dispersion diagrams, it can be used in analyzing more complex, externally excited photonic crystal components or devices, with minimal modifications.

\section{ACKNOWLEDGMENT}

The authors would like to thank D. C. Zografopoulos for providing the MPB results.

\section{REFERENCES}

[1] , K. Busch, S. Lölkes, R. B. Wehrspohn, and H. Föll, Eds., Photonic Crystals: Advances in Design, Fabrication, and Characterization. Weinheim, Germany: Wiley-VCH, 2004.

[2] E. Yablonovitch, "Inhibited spontaneous emission in solid-state physics and electronics," Phys. Rev. Lett., vol. 58, no. 20, pp. 2059-2062, May 1987.

[3] D. Labilloy, H. Benisty, C. Weisbuch, T. F. Krauss, R. M. D. L. Rue, V. Bardinal, R. Houdré, U. Oesterle, D. Cassagne, and C. Jouanin, "Quantitative measurement of transmission, reflection, and diffraction of two-dimensional photonic band gap structures at near-infrared wavelengths," Phys. Rev. Lett., vol. 79, no. 21, pp. 4147-4750, Nov. 1997.

[4] S. G. Johnson, S. Fan, P. R. Villeneuve, and J. D. Joannopoulos, "Guided modes in photonic crystal slabs," Phys. Rev. B, Condens. Matter, vol. 60, no. 8, pp. 5751-5758, Aug. 1999.

[5] S. Kuchinsky, D. C. Allan, N. F. Borelli, and J. C. Cotteverte, "3D localization in a channel waveguide in a photonic crystal with 2D periodicity," Opt. Commun, vol. 175, no. 1, pp. 147-152, Feb. 2000.

[6] M. Lončar, T. Doll, J. Vučković, and A. Scherer, "Design and fabrication of silicon photonic crystal optical waveguides," J. Lightw. Technol., vol. 18, no. 10, pp. 1402-1411, Oct. 2000.

[7] M. Lončar, D. Nedeljković, T. Doll, J. Vučković, A. Scherer, and T. P. Pearsall, "Waveguiding in planar photonic crystals," Appl. Phys. Lett., vol. 77, no. 13, pp. 1937-1939, Sep. 2000.

[8] T. Baba, A. Motegi, T. Iwai, N. Fukaya, Y. Watanabe, and A. Sakai, "Light propagation characteristics of straight single-line-defect waveguides in photonic crystal slabs fabricated into a silicon-on-insulator substrate," IEEE J. Quantum Electron., vol. 38, no. 7, pp. 743-752, Jul. 2002.

[9] S. Fan, S. G. Johnson, and J. D. Joannopoulos, "Waveguide branches in photonic crystals," J. Opt. Soc. Amer. B, Opt. Phys., vol. 18, no. 2 , pp. 162-165, Feb. 2001.

[10] O. Painter, J. Vučković, and A. Scherer, "Defect modes of a two-dimensional photonic crystal in an optically thin dielectric slab," J. Opt. Soc. Amer. B, Opt. Phys., vol. 16, no. 2, pp. 275-285, Feb. 1999.

[11] O. Painter, K. Srinivasan, J. D. O'Brien, A. Scherer, and P. D. Dapkus, "Tailoring of the resonant mode properties of optical nanocavities in two-dimensional photonic crystal slab waveguides," J. Opt. A-Pure Appl. Op., vol. 3, no. 6, pp. 61-70, Oct. 2001.

[12] Y. Akahane, T. Asano, B. S. Song, and S. Noda, "Fine-tuned high-Q photonic-crystal nanocavity," Opt. Express, vol. 13, no. 4, pp. 1202-1214, Feb. 2005.

[13] M. Qiu and M. Swillo, "Contra-directional coupling between two-dimensional photonic crystal waveguides," Photon. Nanostructures, vol. 1, no. 1, pp. 23-30, Dec. 2003.

[14] T. Asano, M. Mochizuki, S. Noda, M. Okano, and M. Imada, "A channel drop filter using a single defect in a 2-D photonic crystal slab-defect engineering with respect to polarization mode and ratio of emissions from upper and lower sides," J. Lightw. Technol., vol. 21, no. 5, pp. 1370-1376, May 2003.

[15] R. D. Meade, A. M. Rappe, K. D. Brommer, and J. D. Joannopoulos, "Nature of the photonic band gap: Some insights from a field analysis," J. Opt. Soc. Amer. B, Opt. Phys., vol. 10, no. 2, pp. 328-332, Feb. 1993.

[16] H. S. Sözüer, J. W. Haus, and R. Inguva, "Photonic bands-convergence problems with the plane-wave method," Phys. Rev. B, Condens. Matter, vol. 45, no. 24, pp. 13962-13972, Jun. 1992.

[17] S. G. Johnson and J. D. Joannopoulos, "Block-iterative frequency-domain methods for Maxwell's equations in a planewave basis," Opt. Express, vol. 8, no. 3, pp. 173-190, Jan. 2001.

[18] C. T. Chan, Q. L. Yu, and K. M. Ho, "Order-N spectral method for electromagnetic waves," Phys. Rev. B, Condens. Matter, vol. 51, no. 23, pp. 16635-16642, Jun. 1995.

[19] A. J. Ward and J. B. Pendry, "Calculating photonic greens functions using a nonorthogonal finite-difference time-domain method," Phys. Rev. B, Condens. Matter, vol. 58, no. 11, pp. 7252-7259, Sep. 1998.

[20] M. Qiu and S. He, "Numerical method for computing defect modes in two-dimensional photonic crystals with dielectric or metallic inclusions," Phys. Rev. B, Condens. Matter, vol. 61, no. 19, pp. 12871-12876, May 2000. 
[21] M. Kafesaki, M. Agio, and C. M. Soukoulis, "Waveguides in finiteheight two-dimensional photonic crystals," J. Opt. Soc. Amer. B, Opt. Phys., vol. 19, no. 9, pp. 2232-2240, Sep. 2002.

[22] M. Kafesaki, C. M. Soukoulis, and M. Agio, "Losses and transmission in two-dimensional slab photonic crystals," J. Appl. Phys., vol. 96, no. 8, pp. 4033-4038, Oct. 2004.

[23] L. C. Andreani and M. Agio, "Photonic bands and gap maps in a photonic crystal slab," IEEE J. Quantum Electron., vol. 38, no. 7, pp. 891-898, Jul. 2002.

[24] M. Koshiba and Y. Tsuji, "Curvilinear hybrid edge/nodal elements with triangular shape for guided-wave problems," J. Lightw. Technol., vol. 18, no. 5, pp. 737-743, May 2000.

[25] V.F. Rodriguez-Esquerre, M. Koshiba, and H. E. Hernandez-Figueroa, "Finite-element analysis of photonic crystal cavities-time and frequency domains," J. Lightw. Technol., vol. 23, no. 3, pp. 1514-1521, Mar. 2005.

[26] M. Vouvakis, Z. Cendes, and J. Lee, "A FEM domain decomposition method for photonic and electromagnetic band gap structures," IEEE Trans. Antennas Propag., vol. 54, no. 2, pp. 721-733, Feb. 2006.

[27] Y. Li and J. Jin, "A vector dual-primal finite element tearing and interconnecting method for solving 3-D large scale electromagnetic problems," IEEE Trans. Antennas Propag., vol. 54, no. 10, pp. 3000-3009, Oct. 2006.

[28] K. Hirayama, M. Kanou, Y. Tsuji, and M. Koshiba, "Curvilinear triangular-prism element for computation of band structure in photonic crystal slab," IEICE Electron. Express, vol. 3, no. 5, pp. 81-86, Mar. 2006.

[29] D. I. Karatzidis, T. V. Yioultsis, and T. D. Tsiboukis, "Gradient-based adjoint variable optimization of broadband microstrip antennas with mixed order prism macroelements," AEUE Int. J. Electron. Commun., vol. 62 , no. 6 , pp. 401-412, 2008.

[30] T. V. Yioultsis and T. D. Tsiboukis, "Development and implementation of second and third order vector finite elements in various 3-D electromagnetic field problems," IEEE Trans. Magn., vol. 33, no. 2, pp. 1812-1815, Mar. 1997.

[31] J. Jin, The Finite Element Method in Electromagnetics. New York: Wiley, 1993.

[32] S. G. Johnson and J. D. Joannopoulos, The MIT Photonic-Bands Package, [Online]. Available: http://ab-initio.mit.edu/mpb/

[33] M. Qiu, "Band gap effects in asymmetric photonic crystal slabs," Phys. Rev. B, Condens. Matter, vol. 66, pp. 331 031-331 034, Jul. 2002.

[34] Meep FDTD Package, [Online]. Available: http://ab-initio.mit.edu/ meep/

[35] S. G. Johnson, P. R. Villeneuve, S. Fan, and J. D. Joannopoulos, "Linear waveguides in photonic crystal slabs," Phys. Rev. B, Condens. Matter, vol. 62, no. 12, pp. 8212-8221, Sep. 2000.

[36] A. Chutinan and S. Noda, "Waveguides and waveguide bends in twodimensional photonic crystal slabs," Phys. Rev. B, Condens. Matter, vol. 62 , no. 7 , pp. $4488-4492$, Aug. 2000.

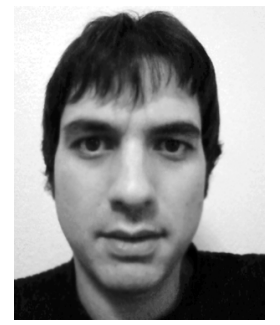

Dimitrios I. Karatzidis was born in Thessaloniki, Greece, in 1974. He received the Diploma degree in electrical and computer engineering from Aristotle University of Thessaloniki (AUTH), Thessaloniki, Greece, in 1999, where he is currently working towards the Ph.D. degree in electrical and computer engineering.

His research interests include computational electromagnetics and optimization.

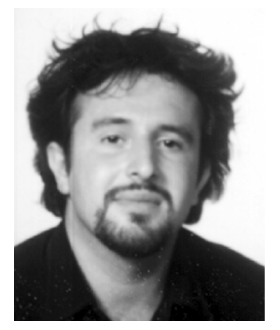

Traianos V. Yioultsis was born in Yiannitsa, Greece, in 1969. He received the Diploma degree (with honors) in electrical engineering and the Ph.D. degree in electrical and computer engineering from the Aristotle University of Thessaloniki (AUTH), Thessaloniki, Greece, in 1992 and 1998, respectively.

From 1993 to 1998, he was a Research and Teaching Assistant in the Department of Electrical and Computer Engineering, AUTH. From 2001 to 2002, he was a Postdoctoral Research Associate at the Department of Electrical and Computer Engineering, University of Illinois at Urbana-Champaign, Urbana. Since 2002, he has been with the Department of Electrical and Computer Engineering, AUTH, where he is currently an Assistant Professor. His current interests include the analysis and design of microwave circuits and antennas with fast computational and optimization techniques and the modeling of complex wave propagation problems.

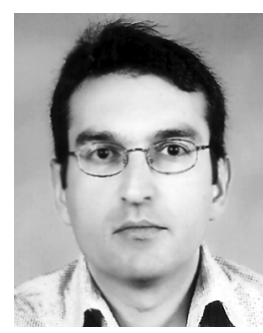

Emmanouil E. Kriezis (S'92-M'96) was born in Thessaloniki, Greece, in 1968. He received the Diploma degree in electrical engineering and the $\mathrm{Ph} . \mathrm{D}$. degree in electrical and computer engineering from the Department of Electrical and Computer Engineering, Aristotle University of Thessaloniki (AUTH), Thessaloniki, Greece, in 1991 and 1996, respectively. His doctoral dissertation focused on the development of full vector beam propagation methods for lightwave propagation in integrated optical devices.

In 1998, he joined the Department of Engineering Science, University of Oxford, Oxford, U.K., initially as an EPSRC Postdoctoral Researcher. In 2001, he was awarded the prestigious Royal Society University Research Fellowship to study light propagation in complex anisotropic media. Since September 2002, he has been Assistant Professor in Optical and Microwave Communications in the Department of Electrical and Computer Engineering, AUTH. His research interests include methods of lightwave propagation, analysis of integrated optical devices, liquid crystal devices, photonic crystals, and photonic crystal fibers.

Dr. Kriezis is a member of the IEEE Lasers and Electro-Optics Society (LEOS). 